\title{
Lipid modification and coronary heart disease in type 2 diabetes: different from the general population?
}

Patients with non-insulin dependent (type 2) diabetes have a 2-5-fold increase in age adjusted mortality from coronary heart disease (CHD). ${ }^{12}$ CHD accounts for up to $70 \%$ of the observed mortality in these patients. ${ }^{12}$ The distinct metabolic background of type 2 diabetes appears to be of pathophysiological importance in producing this excess of $\mathrm{CHD}$, and therefore should provide the key to therapeutic intervention for reducing its incidence.

Type 2 diabetes is characterised by, in addition to hyperglycaemia, raised serum triglycerides and reduced high density lipoprotein (HDL) cholesterol compared to people without diabetes..$^{3-5}$ In contrast, total and low density lipoprotein (LDL) cholesterol concentrations are usually similar to those found in non-diabetic subjects. ${ }^{3-5}$ The importance of this increase in triglycerides is the metabolic link with the production of remnant particles that are highly atherogenic, a reduced HDL cholesterol, and the production of small dense LDL. ${ }^{5}$ It is the small dense LDL species (or LDL-III) of LDL particle distribution that is considered to be particularly atherogenic and is more readily oxidised. Additional features of this "diabetic dyslipidaemia" are increased postprandial lipaemia, compositional changes in very low density lipoprotein (VLDL) and HDL, which are associated with increased coagulant factors-fibrinogen, factor VII, and plasminogen activating factor-I. ${ }^{6}$

There are considerable epidemiological data linking diabetic dyslipidaemia with the incidence of CHD in type 2 diabetes 2 patients. ${ }^{125}$ In the general population a recent meta-analysis has shown that increased serum triglyceride is an independent risk factor for CHD. ${ }^{7}$ However in type 2 diabetes, the strength of the association with serum triglyceride and low HDL cholesterol is stronger and more consistent than for total cholesterol, a distinct difference from that observed in the general population. ${ }^{125}$ This factor, in our opinion, is important in the development of a strategy for therapeutic intervention to reduce the incidence of CHD in type 2 diabetic patients.

With regard to intervention trial data relevant to patients with diabetes, there are several randomised controlled trials of primary (Helsinki heart study, WOSCOPS) and secondary (Stockholm prospective trial, 4S, CARE, LIPID) prevention of CHD with lipid modification. These trials have included only subgroups of diabetic patients as part of the study cohort. Outcome data on clinical coronary events in these diabetic patients, have been fully reported in only one primary prevention and in one secondary prevention trial (Helsinki heart study ${ }^{8}$ and $4 S^{9}$ ), with both studies confirming a 2-3-fold greater incidence of CHD in subjects with diabetes compared to those without.

In the subgroup of men with type 2 diabetes $(n=135)$ from the Helsinki heart study (3.3\% of the total study), ${ }^{8}$ fibrate (gemfibrozil) treated subjects had a $60 \%$ reduction in CHD, although this did not reach significance. Furthermore, for the whole study group there was significantly greater treatment benefit with regard to $\mathrm{CHD}$ reduction

\author{
Glossary \\ CARDS, Collaborative atorvastatin in diabetes study \\ CARE, Cholesterol and recurrent events \\ DAIS, Diabetes atherosclerosis intervention study \\ FIELD, Fenofibrate intervention and event lowering \\ in diabetes trial \\ LDS, Lipids in diabetes study \\ LIPID, Long-term intervention with pravastatin in \\ ischaemic disease \\ 4S, Scandinavian simvastatin survival study \\ SENDCAP, St Mary's Hospital, Ealing, Northwick \\ Park diabetes cardiovascular disease prevention \\ study \\ WOSCOPS, West of Scotland coronary prevention \\ study
}

for those with higher serum triglycerides (with or without low HDL). ${ }^{9}$ Over the five year treatment period with a fibrate there was a $28 \%$ reduction in serum triglycerides and only $11 \%$ reduction in total cholesterol from a baseline mean of $7.5 \mathrm{mmol} / 1$.

The $4 \mathrm{~S}^{10}$ results in the 204 diabetic subjects $(4.4 \%$ of the total group), who were patients with known CHD (postmyocardial infarct or angina pectoris) and a relatively high cholesterol (baseline mean cholesterol $6.7 \mathrm{mmol} / \mathrm{l}$ ), confirmed significant improvements in CHD event rates and mortality at 5.4 years with treatment reductions in serum triglycerides $(-11 \%)$ and total cholesterol $(-27 \%)$, and an increase in HDL cholesterol $(+7 \%)$.

New information comes from the recently published SENDCAP study, ${ }^{11}$ which is the first randomised primary prevention trial of lipid modification specifically in patients with type 2 diabetes. The trial investigated 164 patients (101 male and 63 female) without clinical evidence of CHD, with serum lipid concentrations typical of type 2 diabetes. Patients were randomised to receive, in a double blind fashion, either daily bezafibrate $400 \mathrm{mg}$ or placebo in addition to usual diabetic care. Bezafibrate treatment was associated with significant reductions in serum triglyceride $(-33 \%)$, total cholesterol $(-8 \%)$, and an increase in HDL cholesterol $(+6 \%)$, as well as a trend to reduced fibrinogen $(-18 \% ; \mathrm{p}=0.08)$ at three years. Although no changes in either group were found for the primary end pointultrasonically measured carotid intima medial thickness-in the bezafibrate treated group there was a significant ( $p=0.01, \log$-rank test) reduction in the incidence of "definite CHD events" defined as the combined observation of Minnesota coded probable ischaemic change on the resting ECG and documented myocardial infarction.

In patients without diabetes, clinical trials have established beyond doubt that lowering serum cholesterol can reduce the incidence of new CHD as well as delaying the progression of established disease. We believe that in the routine treatment of patients with type 2 diabetes there 
should be greater emphasis on lipid modification, because of their far greater than average CHD risk, and that this should concentrate on lowering triglyceride and increasing HDL cholesterol, in addition to LDL cholesterol reduction, The data from the Helsinki heart study with a "high cholesterol" study group and SENDCAP with a "normal cholesterol" study group, are consistent with this strategy and form part of the evidence to support this approach.

For secondary prevention, the overall results from the major trials-CARE ${ }^{12}$ and LIPID ${ }^{13}{ }^{14}$ with their larger diabetic subgroups ( $\mathrm{n}=586$ and $\mathrm{n}=777$, respectively)support the strong data from 4 S. However, both these new secondary prevention trials, although with a lower mean baseline cholesterol $(5.7 \mathrm{mmol} / \mathrm{l})$ compared to $4 \mathrm{~S}$, included patients with both type 2 and type 1 diabetes. The latter is a completely different disease, reducing the applicability of these trials to most patients who have type 2 diabetes. The role of treatment to lower triglycerides and increase HDL cholesterol, in addition to cholesterol lowering, in patients with diabetes and manifest CHD needs further study. ${ }^{15} 16$

Dietary modification, weight loss in the overweight, avoidance of alcohol excess, and improved glycaemic control will often reduce serum triglycerides; however, pharmacological intervention is often required. While the statins act mainly to lower total and LDL cholesterol, the fibrates predominately lower triglycerides and increase HDL cholesterol. The statins also lower triglycerides, an effect they all share in proportion to their lowering of LDL cholesterol. ${ }^{17}$ Durrington ${ }^{15}$ has pointed out that the relative decrease in CHD risk for a given decrease in serum cholesterol is greater with fibrates than with statins, suggesting that the additional benefit results from the triglyceride reduction. This may in part be explained by the additional action of fibrates in reducing the proportion of highly atherogenic small dense LDL particles, in favour of those that are larger, less dense, and less atherogenic. ${ }^{18}$

The importance of triglyceride lowering as a key target for lipid modification in patients with type 2 diabetes is now being addressed by investigators who have established studies that are powered sufficiently and incorporate treatments with effective triglyceride lowering. These studies are being conducted specifically in patients with type 2 diabetes: the fibrate trials, using fenofibrate (FIELD, DAIS, and LDS (also in combination with cerivastatin)), and a statin trial (CARDS), using atorvastatin, which also has notable triglyceride lowering properties.

M D FEHER

Section of Clinical Pharmacology,

Imperial College School of Medicine,

Chelsea and Westminster Hospital,

London, UK

Unit for Metabolic Medicine,

R S ELKELES

Imperial College School of Medicine at St Marys' Hospital,

London, UK

1 Pyorala K, Laakso M, Uusitupa M. Diabetes and atherosclerosis: an epidemiologic view. Diabetes Metab Rev 1987;3:463-524.

2 Manson JE, Spelsberg A. Risk modification in the diabetic patient. In: ManManson JE, Spelsberg A. Risk modification in the diabetic patient. In: Man-
son JE, Ridker PM, Gaziano JM, Henekens CH, eds. Prevention of myocarson JE, Ridker PM, Gaziano JM, Henekens CH, eds. Prevent
dial infarction. Oxford: Oxford University Press 1996:241-73.

3 Kannel WB. Lipids, diabetes end coronary heart disease: insights from the Framingham study. Am Heart f 1985;110:1100-7.

4 Howard BV. Lipoprotein metabolism in diabetes mellitus. F Lipid Res 1987; 28:613-28.

5 Syvanne M, Taskinen M-R. Lipids and lipoproteins as coronary risk factors in non-insulin-dependent diabetes mellitus. Lancet 1997;350(suppl 1): $20-3$

6 Jokel R, Colwell JA. Arterial thrombosis in atherosclerosis in diabetes. Diabetes Reviews 1997;5:316-30.

7 Hokanson JE, Austin MA. Plasma triglyceride level is a risk factor for cardiovascular disease independent of high-density lipoprotein cholesterol level: a meta-analysis of population-based prospective studies. 7 Cardiovasc Risk 1996;3:213-19.

8 Koskinen P, Manttari M, Manninen V, et al. Coronary heart disease incidence in NIDDM patients in the Helsinki heart study. Diabetes Care 1992;15:820-5.

9 Manninen V, Tenkanen L, Koskinen P, et al. Joint effects of serum triglyceride and LDL cholesterol and HDL cholesterol concentrations on coronary heart disease risk in the Helsinki heart disease study: implications for treatment. Circulation 1992;85:37-45.

10 Pyorala K, Pedersen TR, Kjekshus J, et al. The Scandinavian simvastatin survival study (4S) group. Diabetes Care 1997;20:614-20.

11 Elkeles RS, Diamond JR, Poulter C, et al. The SENDCAP Study Group. Cardiovascular outcomes in type 2 diabetes. Diabetes Care 1998;21:641-8.

12 Sacks FM, Pfeffer MA, Moye LA, et al. The effect of pravastatin on coronary events after myocardial infarction in patients with average cholesterol levels. N Engl f Med 1996;335:1001-9.

13 The Lipid Study Group. Design features and baseline characteristics of the LIPID (long-term intervention with pravastatin in ischaemic disease) study: a randomised trial in patients with previous acute myocardial infarction and/or unstable angina pectoris. Am $\mathcal{f}$ Cardiol 1995; 76:474-9.

14 Results of the long-term intervention with pravastatin in ischaemic disease (LIPID) [abstract]. American Heart Association Scientific Session, Orlando, Florida, November 1997.

15 Durrington P. Statins and fibrates in the management of diabetic dyslipidaemia. Diabetic Medicine 1997;14:513-16.

16 Feher MD, Foxton J, Banks D, et al. Long-term safety of statin-fibrate combination therapy in the treatment of hypercholesterolaemia in patients with coronary artery disease. Br Heart $\mathcal{F}$ 1995;74:14-17.

7 Stein EA, Lane M, Laskarzewski P. Comparison of statins in hypertriglyceridaemia. Am 7 Cardiol 1998;81:66B-9B.

18 Caslake MJ, Feher MD, Foxton J, et al. Atherogenic lipoprotein phenotype in non-insulin-dependent diabetes; reversal with micronized fenofibrate [abstract]. Diabetic Medicine 1997;14(suppl 1):S41. 